\title{
Dose-Dependent Effect of Estrogen Suppresses the Osteo-Adipogenic Transdifferentiation of Osteoblasts via Canonical Wnt Signaling Pathway
}

\author{
Bo Gao ${ }^{19}$, Qiang Huang ${ }^{19}$, Yan-Shui Lin ${ }^{29}$, Bo-Yuan Wei ${ }^{1}$, Yun-Shan Guo', Zhen Sun ${ }^{1}$, Long Wang ${ }^{1}$, \\ Jing Fan ${ }^{1}$, Hong-Yang Zhang ${ }^{1}$, Yue-Hu Han ${ }^{1}$, Xiao-Jie Li ${ }^{1}$, Jun Shi ${ }^{1}$, Jian Liu ${ }^{1 *}$, Liu Yang ${ }^{1 *}$, Zhuo-Jing Luo ${ }^{1 *}$ \\ 1 Institute of Orthopedic Surgery, Xijing Hospital, Fourth Military Medical University, Xi'an, People's Republic of China, 2 Department of Orthopaedics, First Affiliated \\ Hospital, Chengdu Medical College, Chengdu, People's Republic of China
}

\begin{abstract}
Fat infiltration within marrow cavity is one of multitudinous features of estrogen deficiency, which leads to a decline in bone formation functionality. The origin of this fat is unclear, but one possibility is that it is derived from osteoblasts, which transdifferentiate into adipocytes that produce bone marrow fat. We examined the dose-dependent effect of $17 \beta$-estradiol on the ability of MC3T3-E1 cells and murine bone marrow-derived mesenchymal stem cell (BMMSC)-derived osteoblasts to undergo osteo-adipogenic transdifferentiation. We found that $17 \beta$-estradiol significantly increased alkaline phosphatase activity $(\mathrm{P}<0.05)$; calcium deposition; and Alp, Col1a1, Runx2, and Ocn expression levels dose-dependently. By contrast, $17 \beta-$ estradiol significantly decreased the number and size of lipid droplets, and Fabp4 and PPAR $\gamma$ expression levels during osteoadipogenic transdifferentiation $(\mathrm{P}<0.05)$. Moreover, the expression levels of brown adipocyte markers $(M y f 5$, Elovl3, and Cidea) and undifferentiated adipocyte markers (DIk1, Gata2, and Wnt 10b) were also affected by $17 \beta$-estradiol during osteoadipogenic transdifferentiation. Western blotting and immunostaining further showed that canonical Wnt signaling can be activated by estrogen to exert its inhibitory effect of osteo-adipogenesis. This is the first study to demonstrate the dosedependent effect of $17 \beta$-estradiol on the osteo-adipogenic transdifferentiation of MC3T3-E1 cells and BMMSCs likely via canonical Wnt signaling. In summary, our results indicate that osteo-adipogenic transdifferentiation modulated by canonical Wnt signaling pathway in bone metabolism may be a new explanation for the gradually increased bone marrow fat in estrogen-inefficient condition.
\end{abstract}

Citation: Gao B, Huang Q, Lin Y-S, Wei B-Y, Guo Y-S, et al. (2014) Dose-Dependent Effect of Estrogen Suppresses the Osteo-Adipogenic Transdifferentiation of Osteoblasts via Canonical Wnt Signaling Pathway. PLoS ONE 9(6): e99137. doi:10.1371/journal.pone.0099137

Editor: Zhongjun Zhou, The University of Hong Kong, Hong Kong

Received March 11, 2014; Accepted May 11, 2014; Published June 11, 2014

Copyright: $\odot 2014$ Gao et al. This is an open-access article distributed under the terms of the Creative Commons Attribution License, which permits unrestricted use, distribution, and reproduction in any medium, provided the original author and source are credited.

Funding: This work was supported by the Ministry of Science and Technology of China (2011CB964703), the National High Technology Research and Development Program 863 (2012AA020502), and the China Postdoctoral Science Foundation (2012T50856), and the Program for Changjiang Scholars and Innovative Research Team in University (No. IRT1053). No benefits in any form have been or will be received from a commercial party directly or indirectly by the authors of this manuscript. The funders had no role in study design, data collection and analysis, decision to publish, or preparation of the manuscript.

Competing Interests: The authors have declared that no competing interests exist.

*E-mail: zjluogb@hotmail.com (ZJL); yangliufmmu@gmail.com (LY); liujianhq@sina.com (JL)

9 These authors contributed equally to this work.

\section{Introduction}

The presence of bone marrow fat is indicative of aging and a consequence of osteoporosis, especially in menopausal women [1]. One possible cause of bone marrow fat deposition is the aberrant commitment of bone marrow-derived stem cells (BMMSCs) into adipocytes because of their inability to differentiate into other cell lineages, such as osteoblasts. There exists an inverse relationship between bone marrow fat production and bone formation during osteoporosis, namely, adipogenesis is inhibited in patients with a high bone mass [2,3]. However, the origin of bone marrow fat, the physiological role of adipocytes in bone marrow, and the reasons for the increase in adipogenesis during osteoporosis and the abnormal adipogenic differentiation of BMMSCs are unclear.

In recent years, a correlation has been established between the osteo-adipogenic transdifferentiation of bone marrow cells and numerous bone metabolism diseases. Tuan et al. [4] have shown that hBMMSC-derived osteoblasts, adipocytes, and chondrocytes had the potential to transdifferentiate to each lineage, and these findings provided new insights on the pathogenesis of skeletal diseases such as osteoporosis. Nevertheless, If and to what extent established key factors or signaling pathways of normal osteogenesis and adipogenesis play a role in the transdifferentiation process remains unknown.

Estrogen can regulate several signaling molecules such as Notch, Erk, and Ephrin, which function in bone metabolism $[5,6,7,8]$. Endogenous estrogens also play an important role in the development of bone marrow fat. Not only does uncoupling of the bone remodeling units caused by a decrease in estrogen levels especially after the menopause, there is also a notable increase in adipogenic switch in bone marrow accompanied by a decline in bone mass $[9,10]$. Several studies have shown that estrogen is a negative regulator of adipogenesis [11,12] and essential for osteogenic commitment [13]. For example, estrogen simultaneously induces osteogenesis and inhibits adipogenesis both in vivo and in vitro, which led us to hypothesize that estrogen regulates 
osteo-adipogenic transdifferentiation and increases fat deposition in the osteoporotic marrow.

The wingless-type MMTV integration site (Wnt) signaling pathway plays a fundamental role during embryogenesis and normal cell development. Recent studies in humans have shown mutations in Wnt signaling molecules to result in different diseases $[11,12,13]$. Importantly, Wnt signaling also regulates bone development, adipogenic differentiation, and gene expression in whole process of bone metabolism [14,15]. Specifically, canonical Wnt/ $\beta$-catenin signaling is highly animate in mesenchymal precursor cells and in directing pluripotent cells, especially toward the osteoblast lineage while inhibiting adipogenic differentiation. Canonical Wnt signaling is also involved in the suppression of adipogenesis. For example, canonical Wnt signaling stabilizes and promotes cellular and nuclear $\beta$-catenin levels, which inhibit adipogenesis [16], and the suppression of Wnt signaling is indispensible for $P P A R \gamma$ induction and preadipocyte differentiation [17].

Although estrogen can modulate Wnt signaling directly or indirectly and affect bone metabolism $[18,19,20]$, it is not known if estrogen and Wnt signaling are involved in osteo-adipogenic transdifferentiation. Therefore, we used estrogen-deficient murine in vitro and in vivo models to investigate the effects of estrogen and canonical Wnt signaling on osteo-adipogenic transdifferentiation, as well as to define the underlying mechanism.

\section{Materials and Methods}

\section{Materials}

$\alpha$-Modified minimal essential medium $(\alpha$-MEM) and Dulbecco's modified Eagle's medium (DMEM) were purchased from Thermo Scientific (Beijing, China). Polystyrene culture dishes were obtained from Costar (NY, USA), and fetal bovine serum (FBS) was purchased from Gibco Life Technologies (Grand Island, NY, USA). Anti-GSK-3 $\beta$ (ab32391) and anti- $\beta$-catenin (ab32572) antibodies were purchased from Abcam (Cambridge, USA) and GSK-3 $\beta$ (3D10) mAb (\#9832) was purchased from Cell Signaling (Danvers, MA, USA). Recombinant mouse Wnt-3a (1324-WN002) and DKK-1 (5897-DK-010) were purchased from R\&D Systems (Minneapolis, MN, USA). Penicillin-streptomycin was purchased from Solarbio (Beijing, China). The total RNA extraction kit was purchased from OMEGA. The PrimeScript RT reagent kit and SYBR Premix Ex Taq were purchased from TaKaRa (Dalian, China). The ALP activity assay kit was purchased from GENMED Scientific, Inc. (USA). The BCIP/ NBT alkaline phosphatase color development kit and RIPA buffer were purchased from the Beyotime Institute of Biotechnology (Shanghai, China). Other reagents were of the highest commercial grade and purchased from Sigma Chemical (St. Louis, MO, USA).

\section{Animals and Generation of the Ovariectomized Mice Model}

Healthy female G57BL/6 mice, weighing $20.8 \pm 1.25 \mathrm{~g}$, were obtained from the Experimental Animal Center, Fourth Military Medical University Xi'an, China, and acclimated to laboratory conditions for 1 week before commencement of the experiment. For the OVX experiments, 12-week-old mice were bilaterally ovariectomized. The ovaries of sham-operated mice were left intact. The mice were randomly divided into two groups: sham (sham-operated controls; $\mathrm{n}=12$ ) and OVX (ovariectomized; $\mathrm{n}=12$ ). Both groups of animals were maintained in identical housing conditions that controlled the environment, food, light, and temperature. Ovariectomized mice were allowed to recover for 8 weeks, during which time they developed osteoporosis.

\section{Ethics Statement}

All experimental procedures in animals were approved by the Ethics in Animal Research Committee of the Fourth Military Medical University (permission code 2010C00843).

Table 1. Primer sequences used for RT-PCR analysis.

\begin{tabular}{|c|c|c|}
\hline \multirow{2}{*}{ Gene } & \multicolumn{2}{|l|}{ Sequence } \\
\hline & Forward(5'-3') & Reverse(5'-3') \\
\hline Alp & GAGATGGTATGGGCGTCTC & GTTGGTGTTGTACGTCTTGGA \\
\hline Runx2 & GCACAAACATGGCCAGATTCA & AAGCCATGGTGCCCGTTAG \\
\hline Col1a1 & GACATGTTCAGCTTTGTGGACCTC & GGGACCCTTAGGCCATTGTGTA \\
\hline Ocn & GACAAGTCCCACACAGCAACT & GGACATGAAGGCTTTGTCAGA \\
\hline PPAR 22 & GGAGCCTAAGTTTGAGTTTGCTGTG & TGCAGCAGGTTGTCTTGGATG \\
\hline Fabp4 & TGGGAACCTGGAAGCTTGTCTC & GAATTCCACGCCCAGTTTGA \\
\hline Dlk1 & CTGGACGGTGGCCTCTATGAATG & ATCATCCACGCAGGTGCCTC \\
\hline Gata-2 & GGCTCTACCACAAGATGAATGGA & CGCCATAAGGTGGTGGTTGTC \\
\hline Wnt10b & GGCTGTAACCACGACATGGAC & ACGTTCCATGGCATTTGCAC \\
\hline Lrp5 & CACCATTGATTATGCCGACCAG & TGAGTCAGGCCAAACGGGTAG \\
\hline$D k k-1$ & GCTGCATGAGGCACGCTAT & GCGTTGTGGTCATTACCAAGGTT \\
\hline Gsk3 $\beta$ & GTAGAAACGGAAGCCGCTGAA & AATGGCATAACCCTGTGAAACTGA \\
\hline Myf5 & CAGCCCCACCTCCAACTG & GCAGCACATGCATTTGATACATC \\
\hline Elov/3 & ATGAATTTCTCACGCGGGTTA & GCTTACCCAGTACTCCTCCAAAAA \\
\hline Cidea & CCAGAGTCACCTTCGACCTATACA & CTCGTACATCGTGGCTTTGACA \\
\hline$\beta$-catenin & ATTGATGGAGTTGGACATGG & TGTTCTTGAGTGAAGGACTGA \\
\hline$\beta$-actin & CTGGCACCACACCTTCTACA & GGTACGACCAGAGGCATACA \\
\hline
\end{tabular}



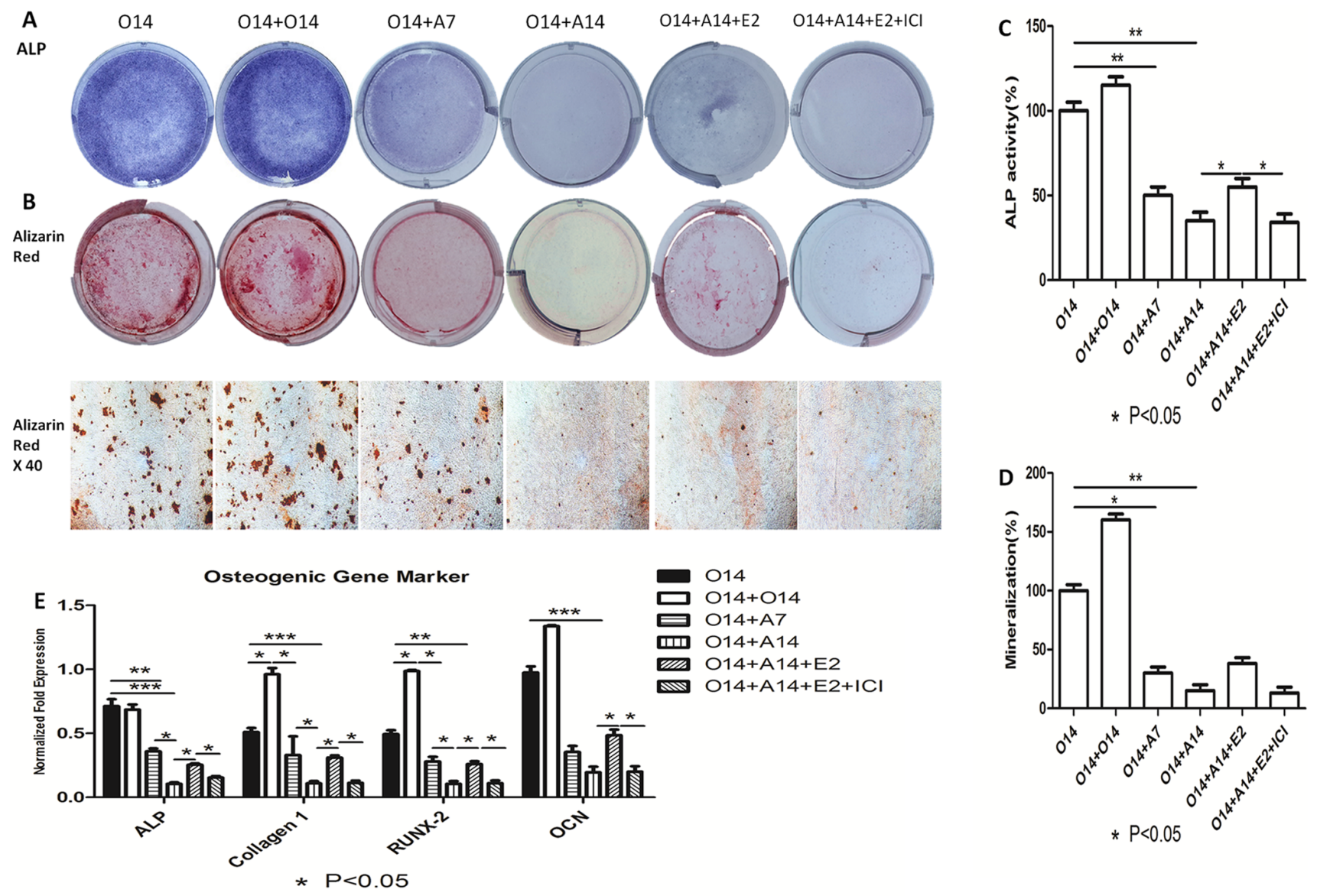

Figure 1. Inhibitory effect of 17beta-estradiol on osteogenic markers of osteo-adipogenic transdifferentiation of MC3T3-E1 cells. After 14 days' osteogenesis, 17beta-estradiol accompanied with or without ICl was added in adipogenic medium for 14 days and 14 days' osteogenesis was used as positive control. 014:14 days' osteogenesis; 014+O14:28 days' osteogenesis; O14+A7:7 days' adipogenesis after 14 days' osteogenesis; O14+A14:14 days' adipogenesis after 14 days' osteogenesis; $014+\mathrm{A} 14+\mathrm{E}_{2}: 14$ days' adipogenesis accompanied with $10^{-7} \mathrm{M}$ of $17 \mathrm{beta-}$ estradiol after 14 days' osteogenesis; $\mathrm{O} 14+\mathrm{A} 14+\mathrm{E}_{2}+\mathrm{ICl}$ : 14 days' adipogenesis accompanied with $10^{-7} \mathrm{M}$ of 17 beta-estradiol and ICl after 14 days' osteogenesis. A: Effect of 17beta-estradiol on the ALP staining of osteo-adipogenic transdifferentiation of MC3T3-E1 cells. B: Effect of 17beta-estradiol on the Alizarin red staining of osteo-adipogenic transdifferentiation of MC3T3-E1 cells. C: Effect of 17beta-estradiol on the ALP activity of osteoadipogenic transdifferentiation of MC3T3-E1 cells. The control value for ALP activity was $0.418 \pm 0.018 \mathrm{unit} / \mathrm{mg}$ protein. D: Effect of $17 \mathrm{beta-estradiol}$ on the mineralization of osteo-adipogenic transdifferentiation of MC3T3-E1 cells. The control value for mineralization was $0.915 \pm 0.020$ OD. E: Effect of 17beta-estradiol on the osteogenic mRNA expression of Alp, Col1a1, Runx2 and Ocn. Expression of each target gene was calculated as a relative expression to beta-actin and represented as normalized fold expression. Data are represented as mean $\pm S D$ of 3 independent experiments. ${ }^{*} P<0.05$ and ${ }^{* *} \mathrm{P}<0.01$.

doi:10.1371/journal.pone.0099137.g001

\section{Cell Culture}

BMMSCs were isolated from sham-operated or OVX C57BL/ 6 mice as previously described [21], and cells were characterized using mesenchymal stem cell minimal criteria [22]. Briefly, the bone marrow was flushed out of long bones of mice and plated as a cell suspension $\left(0.5 \times 10^{6}\right.$ cells $\left.\mathrm{cm}^{-2}\right)$ in $\alpha$-MEM supplemented with $15 \%$ FBS, $2 \mathrm{mM}$ glutamine, $100 \mathrm{U} / \mathrm{ml}$ penicillin, and $100 \mu \mathrm{g} / \mathrm{ml}$ streptomycin. Culture dishes were incubated at $37^{\circ} \mathrm{C}$ in a humidified atmosphere of $95 \%$ air and $5 \% \mathrm{CO}_{2}$. The culture medium was replaced every other day. Homogenous BMMSCs were obtained through three generations.

Murine osteoblastic MC3T3-E1 cells were obtained from the Center Laboratory for Tissue Engineering, College of Stomatology, Fourth Military Medical University, Xi'an, China [23,24]. MC3T3-E1 cells were cultured in $\alpha$-MEM supplemented with $10 \%$ heat-inactivated FBS, $100 \mathrm{U} / \mathrm{ml}$ penicillin, and $100 \mu \mathrm{g} / \mathrm{ml}$ streptomycin at $37^{\circ} \mathrm{C}$ in a humidified atmosphere of $95 \%$ air and $5 \% \mathrm{CO}_{2}$.

\section{Osteoblastic Differentiation and Osteo-adipogenic Transdifferentiation of BMMSCs and MC3T3-E1 Cells}

To induce osteogenesis, BMMSGs or MC3T3-E1 cells were cultured at $1 \times 10^{5}$ cells $/ \mathrm{cm}^{2}$ in DMEM expansion medium supplemented with osteogenic supplements, $1 \mu \mathrm{M}$ dexamethasone, $10 \mathrm{mM} \beta$-glycerophosphate, and $50 \mu \mathrm{g} / \mathrm{ml}$ ascorbic acid for 14 days or 28days.

To induce adipogenic differentiation, cells were cultured at $1 \times 10^{5}$ cells $/ \mathrm{cm}^{2}$ in adipogenic hormonal cocktail expansion medium supplemented with $10 \mu \mathrm{M}$ dexamethasone, $5 \mu \mathrm{g} / \mathrm{ml}$ insulin, and $0.5 \mathrm{mM} 3$-isobutyl-1-methylxantine for 7 or 14 days. The medium was replaced every other day. For transdifferentiation studies, BMMSCs or MC3T3-E1 cells were first cultured in osteogenic medium for 14 or 28 days. Partially or fullydifferentiated osteoblasts were then cultured in adipogenic medium for 7 or 14 days. 

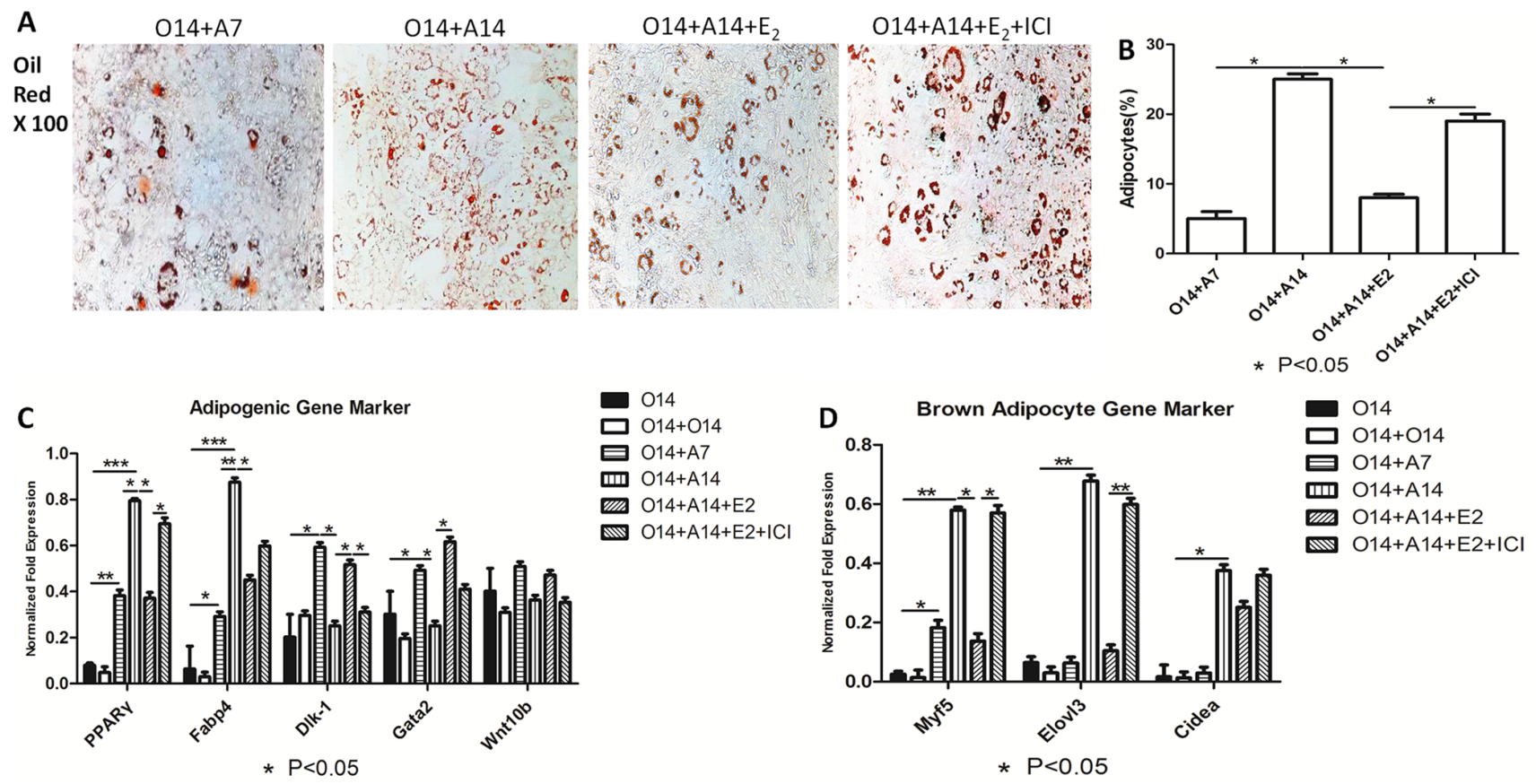

Figure 2. Effect of 17beta-estradiol on adipogenic markers of osteo-adipogenic transdifferentiation of MC3T3-E1 cells. After 14 days' osteogenesis, 17beta-estradiol accompanied with or without ICl was added in adipogenic medium for 14 days and 14 days' osteogenesis was used as positive control. 014:14 days' osteogenesis; 014+014:28 days' osteogenesis; 014+A7:7 days' adipogenesis after 14 days' osteogenesis; 014+A14:14 days' adipogenesis after 14 days' osteogenesis; O14+A14+ $\mathrm{E}_{2}: 14$ days' adipogenesis accompanied with $10^{-7} \mathrm{M}$ of $17 \mathrm{beta-estradiol}$ after 14 days' osteogenesis; $\mathrm{O} 14+\mathrm{A} 14+\mathrm{E}_{2}+\mathrm{ICl}: 14$ days' adipogenesis accompanied with $10^{-7} \mathrm{M}$ of 17 beta-estradiol and ICl after 14 days' osteogenesis. A: Effect of 17beta-estradiol on the Oil red staining of osteo-adipogenic transdifferentiation of MC3T3-E1 cells. B: lipid quantification of each group was valued by the method mentioned in "method and material." C: Effect of 17beta-estradiol on the adipogenic mRNA expression of PPARY, Fabp4, Dlk1, Gata2 and Wnt10b. D: Effect of 17beta-estradiol on brown adipogenic mRNA expression of Myf5, Elov/3 and Cidea. Expression of each target gene was calculated as a relative expression to beta-actin and represented as normalized fold expression. Data are represented as mean \pm SD of 3 independent experiments. ${ }^{*} \mathrm{P}<0.05$ and ${ }^{*} \mathrm{P}<0.01$.

doi:10.1371/journal.pone.0099137.g002

\section{Induction of $17 \beta$-estradiol of BMMSCs and MC3T3-E1 Cells}

To investigate the effect of estrogen on osteo-adipogenic transdifferentiation, MC3T3-E1 cells were treated with $1 \times 10^{-7} \mathrm{M}$ each of $17 \beta$-estradiol and ICI, an estrogen receptor inhibitor. Phenol red-free adipogenic medium was used to eliminate experimental interference. To investigate the dosedependent effect of estrogen on osteo-adipogenic transdifferentiation, OVX-derived BMMSCs were treated with increasing concentrations of $17 \beta$-estradiol $\left(1 \times 10^{-8} \mathrm{M}, \quad 5 \times 10^{-8} \mathrm{M}\right.$, $1 \times 10^{-7} \mathrm{M}, 5 \times 10^{-7} \mathrm{M}$, and $1 \times 10^{-6} \mathrm{M}$ ) for 14 days.

\section{Modulation of Canonical Wnt Signaling in vitro}

To investigate the role of canonical Wnt signaling in the estrogen-induced transdifferentiation of MC3T3-E1 cells, recombinant WNT-3a or DKK-1 protein was added into phenol redfree adipogenic medium supplemented with $1 \times 10^{-7} \mathrm{M}$ each of $17 \beta$-estradiol and ICI. MC3T3-E1 cells were divided into two groups. In the first group, cells were cultured in osteogenic medium for 14 days, followed by an additional 14-day incubation with adipogenic hormonal cocktail medium supplemented with $17 \beta$-estradiol $\left(1 \times 10^{-7} \mathrm{M}\right)$ and DKK-1 $(50 \mathrm{ng} / \mathrm{ml})$, an inhibitor of canonical Wnt signaling. In the second group, cells were cultured in osteogenic medium for 14 days, followed by an additional 14day incubation with adipogenic hormonal cocktail medium supplemented with $1 \times 10^{-7} \mathrm{M}$ each of $17 \beta$-estradiol and ICI, and WNT-3a (50 ng/ml), an activator of canonical Wnt signaling. Western blotting and immunocytochemistry were used to inves- tigate the roles of estrogen and canonical Wnt signaling in the osteo-adipogenic transdifferentiation of MC3T3-E1 cells.

\section{Alkaline Phosphatase (ALP) Staining and Activity Assay}

Cells were washed twice with phosphate-buffered saline (PBS), fixed with $10 \%$ formalin in PBS for $30 \mathrm{~s}$, rinsed with deionized water, and stained with a BCIP/NBT alkaline phosphatase color development kit under protection from direct light. To measure ALP activity, the cells were lysed with RIPA buffer. The samples were then centrifuged at $10,000 \times g$ for 5 min, and ALP activity was measured in clear supernatants with an ALP activity assay kit. Total protein concentrations were determined by the Bradford protein assay. ALP activity was normalized to total protein concentrations.

\section{Alizarin Red Staining for the Mineralized Matrix}

Cells were fixed in ice-cold $10 \%$ formalin for $20 \mathrm{~min}$ and stained with $40 \mathrm{mM}$ alizarin red S ( $\mathrm{pH}$ 4.4, Sigma Chemical) for $45 \mathrm{~min}$ at room temperature. To estimate matrix calcification, the stain was solubilized with $10 \%$ cetylpyridinum chloride by shaking for $15 \mathrm{~min}$. The absorbance of the released alizarin red $\mathrm{S}$ was measured at $562 \mathrm{~nm}$ [25].

\section{Oil red O Staining and Quantification of Adipocytes}

Transdifferentiated adipocyte fat droplets within osteoblasts were observed by the oil red $\mathrm{O}$ staining method with minor modifications [26]. Cell monolayers were fixed in $4 \%$ paraformaldehyde, washed with water, and stained with $0.6 \%(\mathrm{w} / \mathrm{v})$ oil 
A

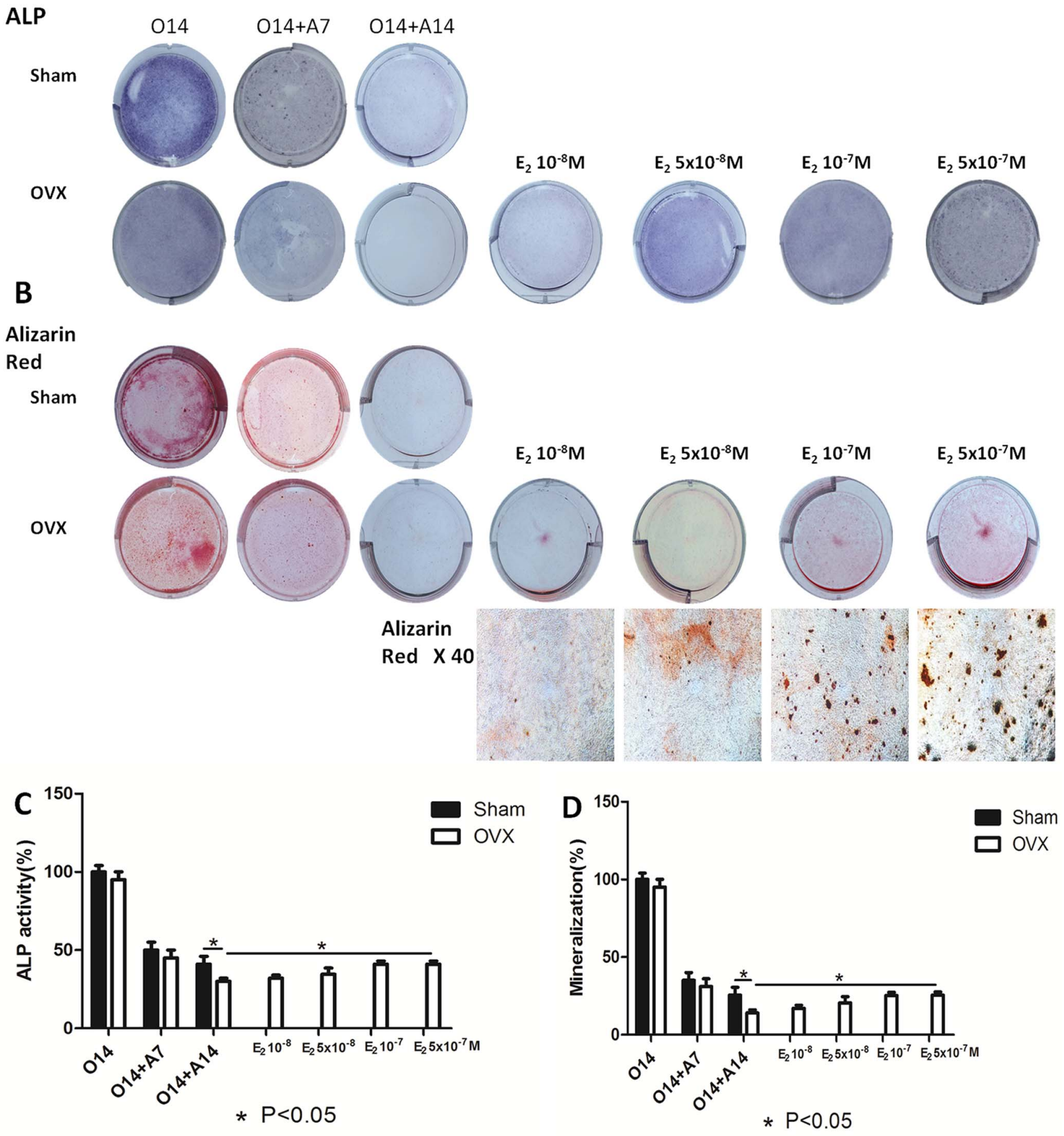

Figure 3. Dose-dependent estrogen on osteogenic markers of osteo-adipogenic transdifferentiation of BMMSCs derived osteoblasts. After 14 days' osteogenesis, different concentrations of 17 beta-estradiol were added in adipogenic medium for 14 days and 14 days' osteogenesis of BMMSCs in OVX group was used as positive control. 014:14 days' osteogenesis; 014+A7:7 days' adipogenesis after 14 days' osteogenesis; O14+A14:14 days' adipogenesis after 14 days' osteogenesis; $E_{2} 10^{-8} \mathrm{M}$ : 14 days' adipogenesis accompanied with $10^{-8} \mathrm{M}$ of $17 \mathrm{beta-}$ estradiol after 14 days' osteogenesis; $\mathrm{E}_{2} 5 \times 10^{-8} \mathrm{M}$ : 14 days' adipogenesis accompanied with $5 \times 10^{-8} \mathrm{M}$ of 17 beta-estradiol after 14 days' osteogenesis; $E_{2} 10^{-7} \mathrm{M}: 14$ days' adipogenesis accompanied with $10^{-7} \mathrm{M}$ of 17 beta-estradiol after 14 days' osteogenesis; $E_{2} 5 \times 10^{-7} \mathrm{M}: 14$ days adipogenesis accompanied with $5 \times 10^{-7} \mathrm{M}$ of 17 beta-estradiol after 14 days' osteogenesis. A: ALP staining of osteo-adipogenic transdifferentiation of BMMSCs. B: ALP activity of osteo-adipogenic transdifferentiation of BMMSCs. The control value for ALP activity was $0.782 \pm 0.019$ unit $/ \mathrm{mg}$ protein. C: Alizarin red staining of osteo-adipogenic transdifferentiation of BMMSCs. D: The mineralization of osteo-adipogenic transdifferentiation of BMMSCs. The control value for mineralization was $0.985 \pm 0.020$ OD. Data are represented as mean \pm SD of 3 independent experiments. ${ }^{*} \mathrm{P}<0.05$ and ${ }^{* *} \mathrm{P}<0.01$. doi:10.1371/journal.pone.0099137.g003

red $\mathrm{O}$ containing $60 \%$ isopropanol for $15 \mathrm{~min}$ at room temperature. For quantification of adipocytes, cell monolayers were washed extensively with water to remove unbound dye, and $1 \mathrm{ml}$ of isopropyl alcohol was added to the culture dish. 


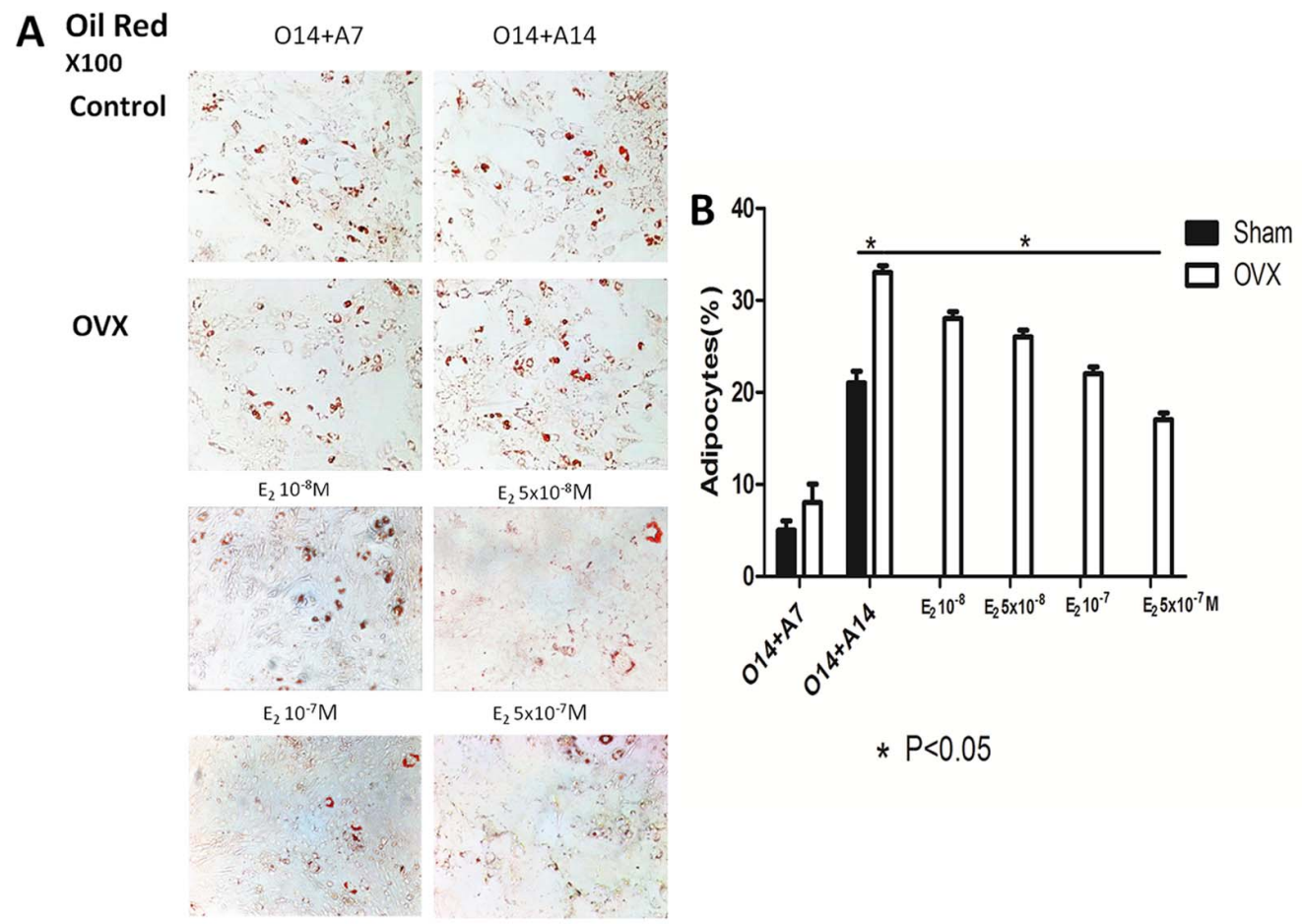

Figure 4. Dose-dependent estrogen on adipogenic markers of osteo-adipogenic transdifferentiation of BMMSCs derived osteoblasts. A: Oil red staining of osteo-adipogenic transdifferentiation of BMMSCs. B: lipid quantification of each group of osteo-adipogenic transdifferentiation of BMMSCs. Data are represented as mean \pm SD of 3 independent experiments. ${ }^{* P}<0.05$ and ${ }^{* * P}<0.01$. doi:10.1371/journal.pone.0099137.g004

Adipocytes were quantified by counting red pixels in five random microscopic images per well using Adobe Photoshop software. Values were expressed as a percentage of the total pixels in each microscopic image [27].

\section{RNA Purification and Quantitative Real-time Polymerase Chain Reaction (qRT-PCR)}

Total RNA was purified from cells using Trizol (Invitrogen). RT-PCR was performed, and results were analyzed as previously described [28]. All RT-PGR experiments were performed in triplicate, and the primers used for PGR experiments are listed in Table 1.

\section{Western Blotting}

Cells were lysed in lysis buffer $(50 \mathrm{mM}$ Tris-HCl pH 7.4, containing $150 \mathrm{mM}$ NaCl, $1 \%$ Nonidet P-40, 0.1\% SDS, $10 \mathrm{mg} /$ $\mathrm{ml}$ leupeptin, $10 \mathrm{mg} / \mathrm{ml}$ pepstatin $\mathrm{A}$, and $10 \mathrm{mg} / \mathrm{ml}$ aprotinin) on ice for $30 \mathrm{~min}$. For western analysis, $300 \mu \mathrm{g}$ of total protein was resolved by $10 \%$ SDS-PAGE, and proteins were transferred onto a PVDF membrane. Anti- $\beta$-catenin $(1: 10000)$ and anti-GSK-3 $\beta$ (1:5000) antibodies were used for immunoblotting. $\beta$-Actin was used as a loading control. The horseradish peroxidase-conjugated secondary antibody was used at a 1:5000 dilution. Images were analyzed by Scion Image software. Immunoreactive bands were quantitatively analyzed in triplicate by normalizing the band intensities to $\beta$-actin on scanned films with Alpha Image software.

\section{Immunostaining}

Cells were fixed in $4 \%$ paraformaldehyde for $15 \mathrm{~min}$, permeabilized with methanol for $10 \mathrm{~min}$, and incubated with anti- $\beta$-catenin (1:250) antibody and anti- GSK-3 $\beta$ (1:250) overnight. On the following day, cells were incubated with a DyLight
594 and 488 secondary antibody (Abcam) for $1 \mathrm{~h}$. After staining nuclei with DAPI $(0.5 \mu \mathrm{g} / \mathrm{ml})$ for $5 \mathrm{~min}$, the cells were analyzed under a FV1000 model confocal microscope (Olympus, Tokyo, Japan). $\beta$-catenin positive cells were counted, and the percentages of cells positive for $\beta$-catenin and cells positive for nuclear $\beta$ catenin were calculated.

\section{Statistical Analysis}

Data were expressed as means \pm S.D. of multiple repeats of the same experiment $(n=5)$. The data for these measurements were analyzed by one-way analysis of variance (ANOVA) with subsequent post hoc multiple comparisons by Dunnett's test. Statistically significant values were defined as $\mathrm{P}<0.05$.

\section{Results}

\section{Estrogen Inhibits the Osteo-adipogenic} Transdifferentiation of MC3T3-E1 Cells

We first investigated the effect of estrogen on the osteoadipogenic transdifferentiation of MC3T3-E1 cells. $10^{-7} \mathrm{M}$ of 17 beta-estradiol was added into adipogenic medium after 14 days' osteogenesis. ALP activity assay, alizarin red and oil red staining (Fig. 1A, B, and Fig. 2A) showed 17 $\beta$-estradiol to suppress osteoadipogenic transdifferentiation. Specifically, the mineralized matrix area of MC3T3-E1 cells cultured in the presence of $17 \beta$ estradiol for 14 days was larger than that of cells cultured in the presence of ICI. 17 $\beta$-Estradiol also affected the morphology of fully-differentiated adipocytes and decreased the number and size of lipid droplets. However, this effect could be neutralized when ICI was used to inhibit the effect of $17 \beta$-estradiol, indicating that estrogen inhibits osteo-adipogenic transdiffentiation. Moreover, ALP activity, calcium deposition, and the quantification of lipid 

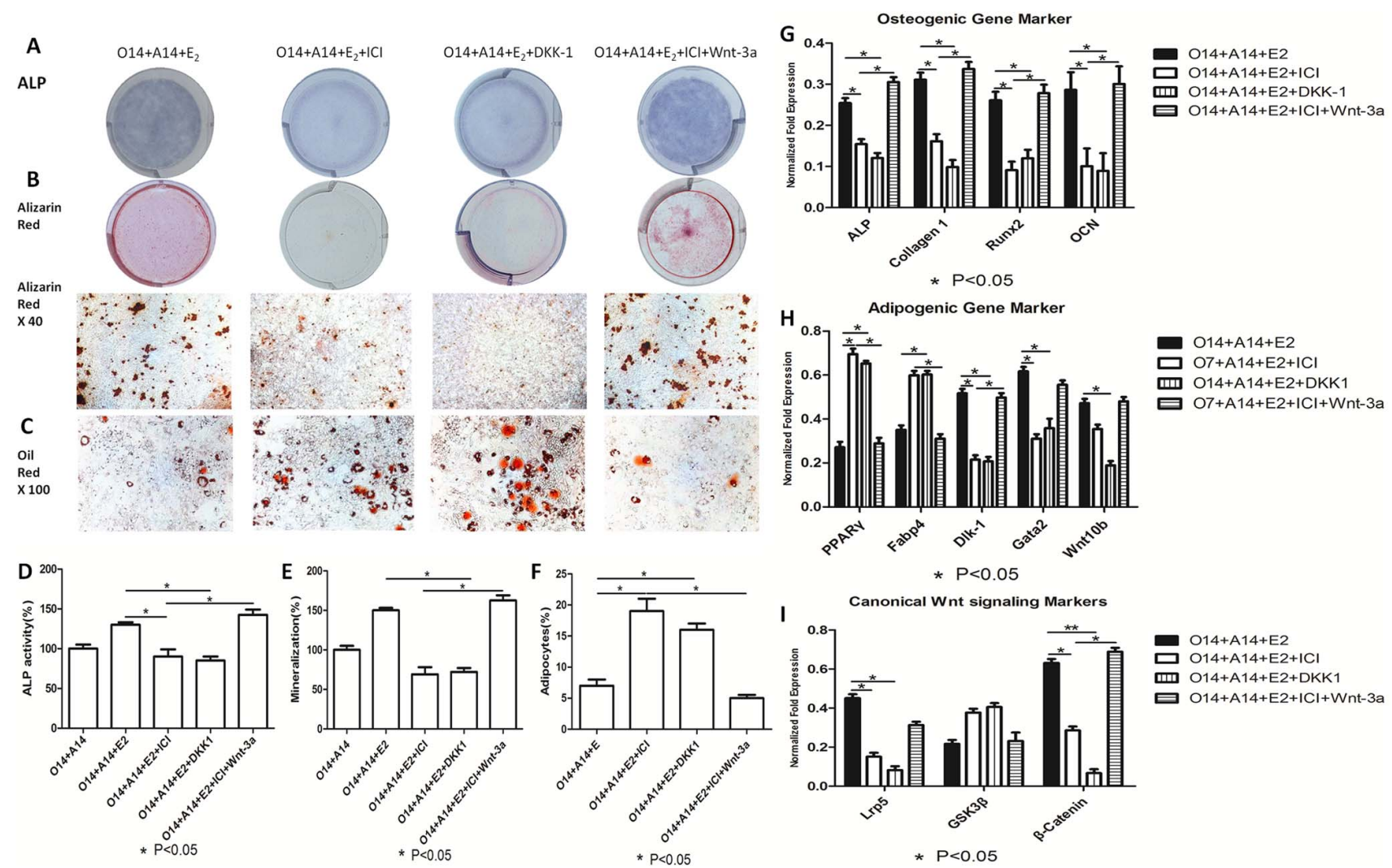

Figure 5. Dose-dependent estrogen inhibits osteo-adipogenic transdifferentiation of MC3T3-E1 cells likely via modulating canonical Wnt singling pathway. After 14 days' osteogenesis, two groups of MC3T3-E1 cells were divided. The first group was cultured in adipogenic cocktail medium accompanied with $10^{-7} \mathrm{M}$ of 17 beta-estradiol for 14 days and $50 \mathrm{ng} / \mathrm{ml}$ DKK-1 was added to block canonical Wnt signaling pathway simultaneously. The second group was cultured in adipogenic cocktail medium accompanied with $10^{-7} \mathrm{M}$ of $17 \mathrm{beta-estradiol}$ and $\mathrm{ICl}$ together for 14 days and $50 \mathrm{ng} / \mathrm{ml}$ Wnt-3a was added to activate canonical Wnt signaling pathway simultaneously. 014+A14:14 days' adipogenesis after 14 days' osteogenesis; $014+\mathrm{A} 14+\mathrm{E}_{2}: 14$ days' adipogenesis accompanied with $10^{-7} \mathrm{M}$ of 17 beta-estradiol after 14 days' osteogenesis; $\mathrm{O} 14+\mathrm{A} 14+\mathrm{E}_{2}+\mathrm{ICl}$ : 14 days' adipogenesis accompanied with $10^{-7} \mathrm{M}$ of 17 beta-estradiol and ICl after 14 days' osteogenesis. O14+A $14+$ $\mathrm{E}_{2}+\mathrm{ICl}+\mathrm{Wnt}-3 \mathrm{a}$ : 14 days' adipogenesis accompanied with $10^{-7} \mathrm{M}$ of 17 beta-estradiol, ICl and $50 \mathrm{ng} / \mathrm{ml} \mathrm{Wnt}-3 \mathrm{a}$ after 14 days' osteogenesis; O14+A14+ $\mathrm{E}_{2}+\mathrm{DKK}-1: 14$ days adipogenesis accompanied with $10^{-7} \mathrm{M}$ 17beta-estradiol and $50 \mathrm{ng} / \mathrm{ml}$ DKK1 after 14 days' osteogenesis. A: ALP staining of osteoadipogenic transdifferentiation of MC3T3-E1 cells; B: Alizarin red staining of osteo-adipogenic transdifferentiation of MC3T3-E1 cells; C: Oil red staining of osteo-adipogenic transdifferentiation of MC3T3-E1 cells; D: ALP activity of osteo-adipogenic transdifferentiation of MC3T3-E1 cells. The control value for ALP activity was $0.687 \pm 0.014$ unit $/ \mathrm{mg}$ protein; $\mathrm{E}$ : The mineralization of osteo-adipogenic transdifferentiation of MC3T3-E1 cells. The control value for mineralization was $0.795 \pm 0.020$ OD; F: Quantification of lipid; G: Effect of Wnts and 17beta-estradiol on the osteogenic mRNA expression; $\mathrm{H}$ : Effect of Wnts and 17beta-estradiol on the adipogenic mRNA expression; I: Effect of Wnts and 17beta-estradiol on the canonical Wnt signaling mRNA expression. Expression of each target gene was calculated as a relative expression to beta-actin and represented as normalized fold expression. Data are represented as mean \pm SD of 3 independent experiments. ${ }^{*} \mathrm{P}<0.05$ and ${ }^{* *} \mathrm{P}<0.01$.

doi:10.1371/journal.pone.0099137.g005

number and size further proved this phenomenon (Fig. 1C, D, and Fig. 2B). In addition, 17ß-estradiol increased (Fig. 1E) and decreased (Fig. 2C) the levels of osteogenic and adipogenic markers, whereas there were no changes in cells simultaneously treated with $17 \beta$-estradiol and ICI. To confirm the delicate changes in osteo-adipogenic transdiffentiation, we examined the expression levels of pre-adipogenic markers, namely, Dlk1, Gata2, and Wnt10b in MC3T3-E1 cells. Figure 2C showed that dedifferentiation preceded the process of transdifferentiation and with the induction of adipogensis getting longer, fully-differentiated osteoblasts regained their ability to multi-differentiate into the adipogenic lineage, whereas fully-differentiated adipocytes dedifferentiated in the presence of $17 \beta$-estradiol. Figure 2D also showed that $17 \beta$-Estradiol downregulated the expression levels of brown adipocyte markers, namely, Myf5, Elovl3, and Cidea, during transdifferentiation, indicating that activation of brown adipogenic genes may be occurring, but at a much later stage in the transdifferentiation process.

\section{Estrogen Dose-dependently Regulates the Osteo- adipogenesis Transdifferentiation of Primary BMMSCs}

To expand the role of $17 \beta$-estradiol in transdifferentiation, we used an ovariectomized C57BL/6 mouse model to investigate the ability of BMMSCs derived osteoblasts to transdifferentiate under osteoporotic conditions. We found the transdifferentiation potential of BMMSCs derived osteoblasts from the OVX group to be higher than that of control cells. In addition, the expression levels of osteogenic markers in BMMSGs derived osteoblasts from the OVX group were lower than those in cells from the control. After 7 and 14 days of adipogenesis, the expression levels of adipogenic markers increased (Fig. S2). By morphological analysis, BMMSCs derived osteoblasts from the OVX group transdifferentiated into the adipogenic lineage more easily than cells from the sham- 
A
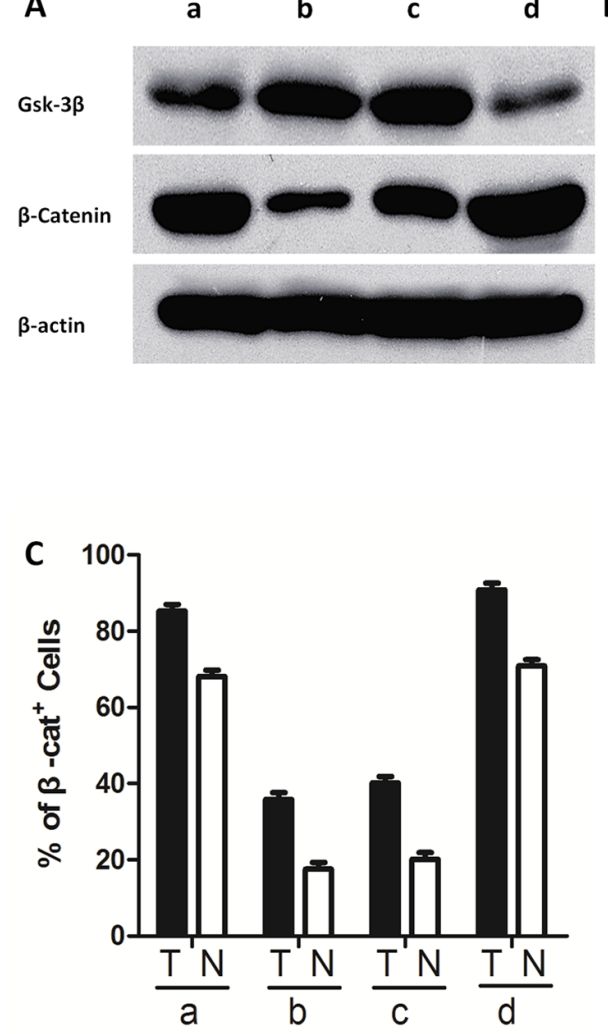

B
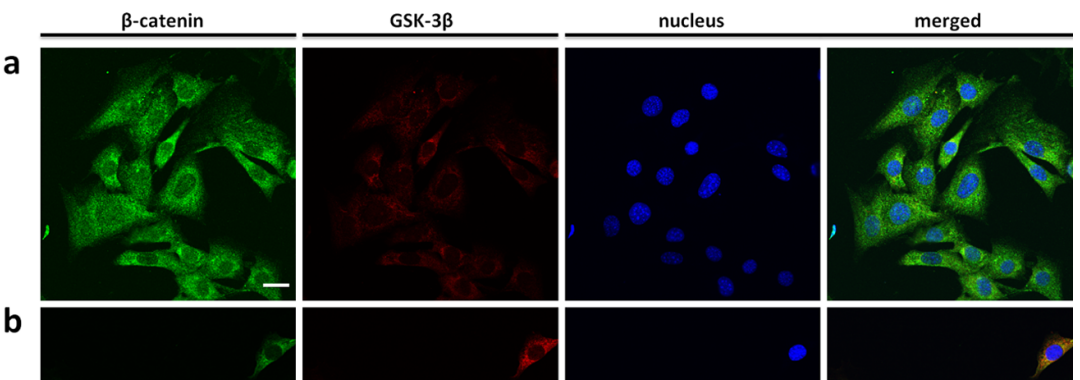

b
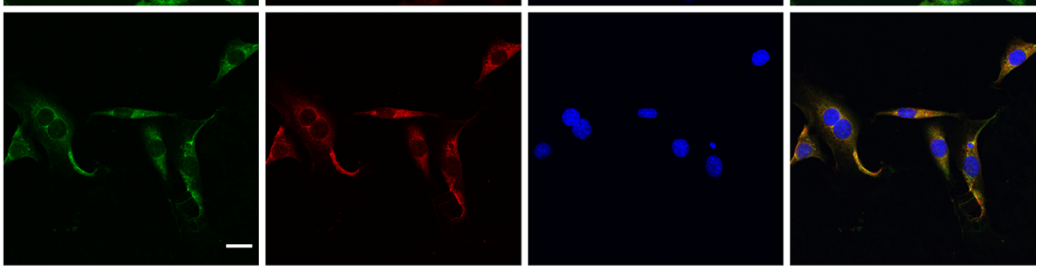

C
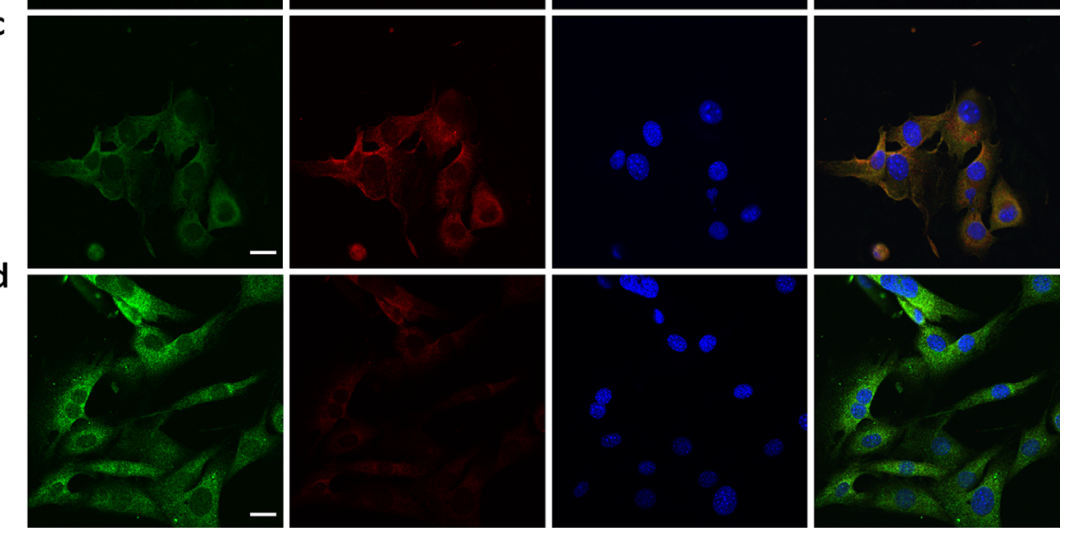

Figure 6. Western blot and immunocytochemistry of canonical Wnt signaling pathway. $a: O 14+A 14+E 2 ; b: 014+A 14+E 2+\mid C l ; c: O 14+A 14+$ E2+DKK-1; d:O14+A14+E2+ICl+Wnt-3a. A: western blot of Gsk3 $\beta$ and $\beta$-catenin; B: Immunocytochemistry of $\beta$-catenin and GSK-3 $\beta$ protein (size bar $=10 \mu \mathrm{m})$. Green: $\beta$-catenin; Red: GSK-3 $\beta$; blue: DAPI staining of nuclei. All photomicrographs were recorded under identical exposure and magnification conditions. C: Percentage of $\beta$-catenin positive cells $(\mathrm{T})$ and cells positive for $\beta$-catenin in the nucleus (N). Data are represented as mean \pm SD of 3 independent experiments.

doi:10.1371/journal.pone.0099137.g006

operated group. ALP activity assay, and alizarin red and oil red staining (Fig. 3A, B, and Fig. 4A) also showed differences in osteoadipogenic transdifferentiation between groups. Specifically, the mineralized matrix area of BMMSCs derived osteoblasts from the OVX group was smaller than that of cells from the control. The morphology of fully-differentiated osteoblasts from the OVX group was also different compared to that of control cells, with a marked increase in the number and size of lipid droplets.

To determine the optimal concentration of $17 \beta$-estradiol for the induction of transdifferentiation, BMMSCs derived osteoblasts were treated with increasing concentrations of the hormone. Our results showed transdifferentiation of BMMSCs derived osteoblasts from the OVX group to decrease with increasing concentration of $17 \beta$-estradiol. This was accompanied by an increase in the mineralized matrix area and a decrease in the number and size of lipid droplets as shown by an ALP activity assay, and alizarin red and oil red staining (Fig. 3A, B, and Fig. 4A). However, there was no difference in the ability of cells treated with $17 \beta$-estradiol at $1 \times 10^{-7} \mathrm{M}$ and $5 \times 10^{-7} \mathrm{M}$ to transdifferentiate by RT-PCR and morphological analysis. These findings were supported by results from the calcium deposition, and the quantification of lipid number and size (Fig. 3C, D and Fig. 4B). To inhibit transdifferentiation, $17 \beta$-estradiol was used at $1 \times 10^{-6}$ and $1 \times 10^{-5} \mathrm{M}$; however, there were no changes compared to $1 \times 10^{-7} \mathrm{M}$. Thus, the potential for osteo-adpogenic transdifferentiation was greater in BMMSGs derived osteoblasts from the OVX group than that of control cells, and with the intervention of optimum concentrations of 17beta-estradiol, the potential got weaker, which further proved the dose-dependent effect of estrogen on the transdifferentiation process.

\section{Estrogen Suppresses Osteo-adipogenic \\ Transdifferentiation via Canonical Wnt Signaling}

To understand the role of canonical Wnt signaling in osteoadipogenic transdifferentiation, we used recombinant WNT-3a and DKK-1 proteins to activate and inhibit this pathway, respectively. We found significant increases in ALP staining and activity; calcium deposition; and Alp, Col1a1, Runx2, and Ocn expression levels in cells treated with WNT-3a, 17 $\beta$-estradiol, and ICI $(\mathrm{P}<0.05)$ (Fig. 5G). However, there was a decrease in the expression levels of bone formation markers when cells were treated with $17 \beta$-estradiol and DKK-1 (Fig. 5A, B, D, and E). The co-incubation of cells with $17 \beta$-estradiol and DKK-1 also increased the number and size of lipid droplets (Fig. 5C, F) and reduced Fabp4, PPAR, Dlk1, Gata2, and Wnt10b expression levels (Fig. $5 \mathrm{H}$ ). By contrast, the incubation of cells with $17 \beta$-estradiol, WNT-3a, and ICI decreased the expression levels of adipogenic markers. Furthermore, western blotting and immunocytochemistry showed canonical Wnt signaling to affect osteo-adipogeic transdifferentiation in the presence of estrogen and ICI. Specifically, Western blot showed that Wnt-3a accompanied with $10^{-7} \mathrm{M}$ of 17 beta-estradiol and ICI increased $\beta$-catenin expression level, while reduced the expression of GSK-3 $\beta$ level. On the 
other hand, DKK-1 decreased the elevated $\beta$-catenin level and the GSK-3 $\beta$ level, which was modulated by $1 \times 10^{-7} \mathrm{M}$ of $17 \beta$ estradiol (Fig. 6A). Moreover, immunostaining showed that WNT$3 \mathrm{a}, 17 \beta$-estradiol, and ICI increased the levels of total and the relative nuclear $\beta$-catenin while decreased the level of total Gsk$3 \beta$, whereas DKK-1 inhibited the effect induced by $10^{-7} \mathrm{M}$ of $17 \beta$-estradiol (Fig. 6B, C).

From the data presented above, we firmly believed that dosedependent estrogen can inhibit the osteo-adipogenic transdifferention effect of MC3T3-E1 cells and murine BMMSGs derived osteoblasts via partially or maybe dependently controlling canonical Wnt signaling pathway.

\section{Discussion}

This study showed for the first time the dose-responsive effect of $17 \beta$-estradiol on the ability of MC3T3-E1 cells and murine BMMSC-derived osteoblasts to transdifferentiate into adipocytes likely via the canonical Wnt signaling pathway. Transdifferentiation is the irreversible switching of differentiated cells that sometimes occurs during disease [29]. It occurs when partially differentiated cells (e.g., pre-osteoblasts) switch to another lineage (e.g., adipocytes) [30]. This phenomenon can occur between committed or differentiated osteoblasts and adipocytes, and it has even been reported to occur at the single cell level [31,32,33,34].

The adipogenic transdifferentiation potential of MC3T3-E1 cells has been previously shown by the ectopic expression of adipogenic transcription factors, namely, $P P A R \gamma, C / E B P \alpha$, or both [35]. Moreover, several factors can regulate osteo-adipogenic transdifferentiation, which is consistent with our finding that $17 \beta$ estradiol can inhibit the osteo-adipogenic transdifferentiation of MC3T3-E1 cells. In this study, we used time-dependent in vitro transdifferentiation models and reported that MC3T3-E1 cells underwent osteo-adipogenic transdifferentiation under proper conditions. With increasing time after osteogenic induction, the osteo-adipogenic transdifferentiation potential decreased. Specifically, the transdifferentiation potential of 3- and 7-day-old osteogenic MC3T3-E1 cells was higher than that of 14- and 21day-old cells (Fig. S1). However, 3- and 7- day-old osteogenic MC3T3-E1 cells did not fully differentiate into mature osteoblasts. Therefore, the osteo-adipogenic transdifferentiation effect of them can hardly win applause. Moreover, the osteo-adipogenic transdifferentiation potential of 21-day-old osteogenic MC3T3E1 cells was hardly observed both by RT-PCR and morphological analysis. Therefore, transdifferentiation was studied in 14-day-old osteogenic adipocytes.

Estrogen can regulate the differentiation potential of BMMSCs into the osteoblast or adipocyte lineage. Recent studies have shown a correlation between a decreased estrogen level after menopause and a marked increase in bone marrow adipogenesis $[9,10]$. In light of these findings, we used an ovariectomized mouse model to investigate the effects of $17 \beta$-estradiol on transdifferentiation. We found the transdifferentiation potential to be higher in BMMSCs derived osteoblasts from the OVX group than that of control cells. In addition, increasing concentrations of $17 \beta$ estradiol could partially or completely reverse osteo-adipogenic transdifferentiation in BMMSCs derived osteoblasts, illustrating the dose-dependent effects of estrogen. Although estrogen has been shown to inhibit and promote adipogenesis and osteogenesis both in vivo and in vitro, respectively [9,36,37], this is the first study to demonstrate the dose-dependent effect of estrogen on the osteoadipogenic transdifferentiation of MC3T3-E1 cells and murine BMMSCs derived osteoblasts and further efforts should be made urgently to clarify the detailed mechanism among the magic transdifferentiation process.

Santanam et al. showed that an in vitro $\mathrm{E}_{2}$ concentration of $10^{-9}$ to $10^{-8} \mathrm{M}$ is generally regarded as a physiologic concentration. Although men have lower estrogen level compared to women, the level may be still higher than the physiologic level which is enough to maintain bone mass in vivo [38]. Moreover, the mechanism of osteoporosis is very complex and estrogen only functions as one part of the metabolism process. There are still many other factors which control the bone mass such as androgens [39], PTH [40], mechanical stress [41], diet [42] and metabolism of calcium and phosphorous [43]. And these factors might compensate the role of low-level estrogen on bone metabolism of men.

Having established that MC3T3-E1 cells and BMMSC-derived osteoblasts transdifferentiated into adipocytes, the expression levels of several brown fat-specific markers were examined to determine whether the cells being formed were white or brown adipocytes. Recent findings from lineage tracing studies have shown that brown adipocytes develop in vivo from a myf5-positive progenitor cell [44], suggesting that myf5-expressing MC3T3-E1 cells and BMMSCs might develop into brown adipocytes. In this study, myf5 expression in MC3T3-E1 cells and BMMSCs was upregulated when cells were cultured in adipogenic medium. Other brown adipocyte-specific markers such as Elovl3 and Cidea were also activated in cells cultured in adipogenic medium, but not until day 7 of adipogenic differentiation, and their expression was more variable. These results demonstrate that activation of brown adipogenic genes may be occurring, but at a later stage in the transdifferentiation process, for which further investigation is required. Although $17 \beta$-estradiol failed to upregulate brown fat genes, our results indicate that the dose-dependent changes in lipid accumulation correlate with white rather than brown adipogenesis.

We also found that osteo-adipogenic transdifferention upregulated adipogenic markers such as PPAR and Fabp4, but downregulated osteogenic markers such as Alp, Runx2, Colla1, and Ocn. Moreover, the expression levels of Dlk1, Gata2, and Wnt10b, which are highly expressed in undifferentiated preadipocytes, were induced. These results indicate that transdifferentiation process combines the effect of transdifferentiation, dedifferentiation, proliferation and differentiation together. Specifically, the dedifferentiation effect was indeed happened prior to the transdifferentiation, and with the induction of adipogenesis getting longer, fully-differentiated osteoblasts regained the multi-differentiation potential and then differentiated to adipogenic lineage. What's more, under the intervention of $17 \beta$-estradiol, fullydifferentiated mature adipocyte could be dedifferentiating again.

Gustafson et al. [45] have shown that Wnt signaling in mature adipocytes increases the $\beta$-catenin level, resulting in the dedifferentiation of 3T3L1 pre-adipocyte cells, which may have been modulated by canonical Wnt signaling $[46,47,48]$. Activation of canonical Wnt signaling has been reported to inhibit adipogenesis [16], and inhibition of Wnt signaling, especially WNT-3a, is essential for $P P A R \gamma$ upregulation and preadipocyte differentiation [17]. These findings are in line with previous studies that show the involvement of estrogen and canonical Wnt signaling in bone metabolism $[18,19,20]$. Luis et al. have also demonstrated that the transdifferentiation of human osteoblasts into adipocytes increased after treatment with fulvestrant, an estrogen receptor blocker. They also suspected the transdifferentiation process might be related to down-regulation of $\beta$-catenin [49]. These inspired us about the mechanism of Wnt signaling on the transdifferentiation 
of MC3T3-E1 cells and primary murine BMMSCs derived osteoblasts under estrogen control.

We used MC3T3-E1 cells to investigate the roles of estrogen and canonical Wnt signaling in osteo-adipogenic transdifferentiation. $17 \beta$-estradiol dose-dependently inhibited the transdifferentiation of MC3T3-E1 cells via canonical Wnt signaling partially or dependently. RT-PCR, western blotting, and immunocytochemistry results showed that WNT-3a activated canonical Wnt signaling and inhibited osteo-adipogenic transdifferentiation independent of $17 \beta$-estradiol and ICI. DKK-1 also blocked canonical Wnt signaling to relieve the inhibition of the osteo-adipogenic transdifferentiation from the presence of $17 \beta$-estradiol. These data indicate that canonical Wnt signaling might act downstream of estrogen to regulate osteo-adipogenic transdifferentiation. These results also support the plasticity of MC3T3-E1 cells and BMMSC-derived osteoblasts, and they suggest that these cells can be used for cell engineering. In light of the findings that canonical Wnt signaling induces the dedifferentiation of fullydifferentiated adipocytes and inhibits the transdifferentiation of osteoblasts, it is possible that perturbations in this signaling cascade may result in bone metabolism diseases such as osteoporosis.

Given the widespread prevalence of estrogen deficiency, especially in post-menopausal women, it is important to understand the role of this hormone in osteo-adipogenic transdifferentiation. Moreover, the involvement of canonical Wnt signaling in this cellular process is likely to provide further insights on the pathogenesis of osteoporosis.

\section{Supporting Information}

Figure S1 Effect of different time-point of the osteoadipogenic transdifferentiation of MC3T3-E1 cells. MC3T3-E1 cells were divided with two groups. Group 1: Cells were first treated in osteogenic medium for 3, 7, 14, or 21 days and then cultured in control medium for 14 days. Group 2: Gells were first treated in osteogenic medium for $3,7,14$, or 21 days and then cultured in adipogenic cocktail medium for 14 days osteoadipogenic transdifferentiation. O3C14 or O3A14:3 days' osteogenesis and then cultured in control or adipogenic cocktail medium for 14 days; O7C14 or O7A14:7 days' osteogenesis and

\section{References}

1. Menagh PJ, Turner RT, Jump DB, Wong CP, Lowry MB, et al. (2010) Growth hormone regulates the balance between bone formation and bone marrow adiposity. J Bone Miner Res 25: 757-768.

2. Verma S, Rajaratnam JH, Denton J, Hoyland JA, Byers RJ (2002) Adipocytic proportion of bone marrow is inversely related to bone formation in osteoporosis. J Clin Pathol 55: 693-698.

3. Qiu W, Andersen TE, Bollerslev J, Mandrup S, Abdallah BM, et al. (2007) Patients with high bone mass phenotype exhibit enhanced osteoblast differentiation and inhibition of adipogenesis of human mesenchymal stem cells. J Bone Miner Res 22: 1720-1731.

4. Song L, Tuan RS (2004) Transdifferentiation potential of human mesenchymal stem cells derived from bone marrow. FASEB J 18: 980-982.

5. Abdallah BM, Ditzel N, Mahmood A, Isa A, Traustadottir GA, et al. (2011) DLK1 is a novel regulator of bone mass that mediates estrogen deficiencyinduced bone loss in mice. J Bone Miner Res 26: 1457-1471.

6. Kamiya Y, Chen J, Xu M, Utreja A, Choi T, et al. (2013) Increased mandibular condylar growth in mice with estrogen receptor beta deficiency. J Bone Miner Res 28: 1127-1134.

7. Song L, Zhao J, Zhang X, Li H, Zhou Y (2013) Icarïn induces osteoblast proliferation, differentiation and mineralization through estrogen receptormediated ERK and JNK signal activation. Eur J Pharmacol 714: 15-22.

8. Pierroz DD, Rufo A, Bianchi EN, Glatt V, Capulli M, et al. (2009) BetaArrestin2 regulates RANKL and ephrins gene expression in response to bone remodeling in mice. J Bone Miner Res 24: 775-784.

9. Justesen J, Stenderup K, Ebbesen EN, Mosekilde L, Steiniche T, et al. (2001) Adipocyte tissue volume in bone marrow is increased with aging and in patients with osteoporosis. Biogerontology 2: 165-171. then cultured in control or adipogenic cocktail medium for 14 days. O14C14 or O14A14:14 days' osteogenesis and then cultured in control or adipogenic cocktail medium for 14 days. O21G14 or O21A14:21 days osteogenesis and then cultured in control or adipogenic cocktail medium for 14 days. Expression of each target gene was calculated as a relative expression to beta-actin and represented as normalized fold expression. Data are represented as mean $\pm \mathrm{SD}$ of 3 independent experiments. $* \mathrm{P}<0.05$ and $* * \mathrm{P}<$ 0.01 .

(TIF)

Figure S2 Dose-dependent estrogen on osteo-adipogenic transdifferentiation of primary murine BMMSGs derived osteoblasts. After 14 days' osteogenesis, different concentrations of 17 beta-estradiol were added in adipogenic medium for 14 days and 14 days' osteogenesis of BMMSCs in OVX group was used as positive control. O14:14 days' osteogenesis; O14+A7:7 days' adipogenesis after 14 days' osteogenesis; O14+A14:14 days' adipogenesis after 14 days' osteogenesis; $\mathrm{E}_{2} 10^{-8} \mathrm{M}$ : 14 days' adipogenesis accompanied with $10^{-8} \mathrm{M}$ of 17 beta-estradiol after 14 days' osteogenesis; $\mathrm{E}_{2} 5 \times 10^{-8}$ M: 14 days' adipogenesis accompanied with $5 \times 10^{-8} \mathrm{M}$ of 17 betaestradiol after 14 days' osteogenesis; $\mathrm{E}_{2} 10^{-7} \mathrm{M}$ : 14 days' adipogenesis accompanied with $10^{-7} \mathrm{M}$ of 17 beta-estradiol after 14 days' osteogenesis; $\mathrm{E}_{2} 5 \times 10^{-7} \mathrm{M}$ : 14 days' adipogenesis accompanied with $5 \times 10^{-7} \mathrm{M}$ of 17 beta-estradiol after 14 days' osteogenesis. Effect of 17 beta-estradiol on the mRNA expression of Alp, Col1a1, Ocn, PPAR , Fabp4, Dlk1 and Myf5. Expression of each target gene was calculated as a relative expression to betaactin and represented as normalized fold expression. Data are represented as mean $\pm \mathrm{SD}$ of 3 independent experiments. ${ }^{*} \mathrm{P}<0.05$ and $* * \mathrm{P}<0.01$

(TIF)

\section{Author Contributions}

Conceived and designed the experiments: BG LY ZJL. Performed the experiments: BG QH YSL BYW HYZ ZS. Analyzed the data: YSG XJL YHH JS. Contributed reagents/materials/analysis tools: JF JL LW. Wrote the paper: BG QH.

10. Gambacciani M, Ciaponi M, Cappagli B, Piaggesi L, De Simone L, et al. (1997) Body weight, body fat distribution, and hormonal replacement therapy in early postmenopausal women. J Clin Endocrinol Metab 82: 414 417.

11. Guo YF, Xiong DH, Shen H, Zhao LJ, Xiao P, et al. (2006) Polymorphisms of the low-density lipoprotein receptor-related protein 5 (LRP5) gene are associated with obesity phenotypes in a large family-based association study. J Med Genet 43: 798-803.

12. Mani A, Radhakrishnan J, Wang H, Mani A, Mani MA, et al. (2007) LRP6 mutation in a family with early coronary disease and metabolic risk factors. Science 315: 1278-1282.

13. Grant SF, Thorleifsson G, Reynisdottir I, Benediktsson R, Manolescu A, et al. (2006) Variant of transcription factor 7-like 2 (TCF7L2) gene confers risk of type 2 diabetes. Nat Genet 38: 320-323.

14. Gong Y, Slee RB, Fukai N, Rawadi G, Roman-Roman S, et al. (2001) LDL receptor-related protein 5 (LRP5) affects bone accrual and eye development. Cell 107: 513-523

15. Kato M, Patel MS, Levasseur R, Lobov I, Chang BH, et al. (2002) Cbfalindependent decrease in osteoblast proliferation, osteopenia, and persistent embryonic eye vascularization in mice deficient in Lrp5, a Wnt coreceptor. J Cell Biol 157: 303-314.

16. Krishnan V, Bryant HU, Macdougald OA (2006) Regulation of bone mass by Wnt signaling. J Clin Invest 116: 1202-1209.

17. Qiu W, Chen L, Kassem M (2011) Activation of non-canonical Wnt/JNK pathway by Wnt3a is associated with differentiation fate determination of human bone marrow stromal (mesenchymal) stem cells. Biochem Biophys Res Commun 413: 98-104.

18. Rossini M, Gatti D, Adami S (2013) Involvement of WNT/beta-catenin signaling in the treatment of osteoporosis. Calcif Tissue Int 93: 121-132. 
19. Sadie-Van GH, Crowther NJ, Hough FS, Ferris WF (2013) The interrelationship between bone and fat: from cellular see-saw to endocrine reciprocity. Cell Mol Life Sci 70: 2331-2349.

20. Kim MH, Park JS, Seo MS, Jung JW, Lee YS, et al. (2010) Genistein and daidzein repress adipogenic differentiation of human adipose tissue-derived mesenchymal stem cells via Wnt/beta-catenin signalling or lipolysis. Cell Prolif 43: $594-605$.

21. Bouffi C, Bony C, Courties G, Jorgensen C, Noel D (2010) IL-6-dependent PGE2 secretion by mesenchymal stem cells inhibits local inflammation in experimental arthritis. PLoS One 5: e14247.

22. Dominici M, Le Blanc K, Mueller I, Slaper-Cortenbach I, Marini F, et al. (2006) Minimal criteria for defining multipotent mesenchymal stromal cells. The International Society for Cellular Therapy position statement. Cytotherapy 8: 315-317.

23. Li Y, Tang L, Duan Y, Ding Y (2010) Upregulation of MMP-13 and TIMP-1 expression in response to mechanical strain in MC3T3-E1 osteoblastic cells. BMC Res Notes 3: 309.

24. Li Y, Ma W, Feng Z, Wang Z, Zha N, et al. (2013) Effects of irradiation on osteoblast-like cells on different titanium surfaces in vitro. J Biomed Mater Res B Appl Biomater 101: 9-17.

25. Stanford CM, Jacobson PA, Eanes ED, Lembke LA, Midura RJ (1995) Rapidly forming apatitic mineral in an osteoblastic cell line (UMR 106-01 BSP). J Biol Chem 270: 9420-9428.

26. Kelly KA, Gimble JM (1998) 1,25-Dihydroxy vitamin D3 inhibits adipocyte differentiation and gene expression in murine bone marrow stromal cell clones and primary cultures. Endocrinology 139: 2622-2628.

27. Taipaleenmaki H, Abdallah BM, AlDahmash A, Saamanen AM, Kassem M (2011) Wnt signalling mediates the cross-talk between bone marrow derived preadipocytic and pre-osteoblastic cell populations. Exp Cell Res 317: 745-756.

28. Zhang JK, Yang L, Meng GL, Yuan Z, Fan J, et al. (2013) Protection by salidroside against bone loss via inhibition of oxidative stress and bone-resorbing mediators. PLoS One 8: e57251.

29. Burke ZD, Tosh D (2005) Therapeutic potential of transdifferentiated cells. Clin Sci (Lond) 108: 309-321.

30. Schilling T, Kuffner R, Klein-Hitpass L, Zimmer R, Jakob F, et al. (2008) Microarray analyses of transdifferentiated mesenchymal stem cells. J Cell Biochem 103: 413-433.

31. Nuttall ME, Patton AJ, Olivera DL, Nadeau DP, Gowen M (1998) Human trabecular bone cells are able to express both osteoblastic and adipocytic phenotype: implications for osteopenic disorders. J Bone Miner Res 13: 371382.

32. Park SR, Oreffo RO, Triffitt JT (1999) Interconversion potential of cloned human marrow adipocytes in vitro. Bone 24: 549-554.

33. Schiller PC, D'Ippolito G, Brambilla R, Roos BA, Howard GA (2001) Inhibition of gap-junctional communication induces the trans-differentiation of osteoblasts to an adipocytic phenotype in vitro. J Biol Chem 276: 14133-14138.
34. Schilling T, Noth U, Klein-Hitpass L, Jakob F, Schutze N (2007) Plasticity in adipogenesis and osteogenesis of human mesenchymal stem cells. Mol Cell Endocrinol 271: 1-17.

35. Kim SW, Her SJ, Kim SY, Shin CS (2005) Ectopic overexpression of adipogenic transcription factors induces transdifferentiation of MC3T3-E1 osteoblasts. Biochem Biophys Res Commun 327: 811-819.

36. Heim M, Frank O, Kampmann G, Sochocky N, Pennimpede T, et al. (2004) The phytoestrogen genistein enhances osteogenesis and represses adipogenic differentiation of human primary bone marrow stromal cells. Endocrinology 145: 848-859.

37. Heine PA, Taylor JA, Iwamoto GA, Lubahn DB, Cooke PS (2000) Increased adipose tissue in male and female estrogen receptor-alpha knockout mice. Proc Natl Acad Sci U S A 97: 12729-12734.

38. Santanam N, Shern-Brewer R, McClatchey R, Castellano PZ, Murphy AA, et al. (1998) Estradiol as an antioxidant: incompatible with its physiological concentrations and function. J Lipid Res 39: 2111-2118.

39. Ebeling PR (2010) Androgens and osteoporosis. Curr Opin Endocrinol Diabetes Obes 17: 284-292

40. McNeilly T, McNally C, Finch M, Beringer T (2013) Recombinant PTH: a study of the outcome of teriparatide therapy for 138 patients with osteoporosis. Ulster Med J 82: 89-93.

41. Ratti C, Vulcano E, Canton G, Marano M, Murena L, et al. (2013) Factors affecting bone strength other than osteoporosis. Aging Clin Exp Res 25 Suppl 1: S9-S11.

42. Govindarajan P, Bocker W, El KT, Kampschulte M, Schlewitz G, et al. (2014) Bone matrix, cellularity, and structural changes in a rat model with highturnover osteoporosis induced by combined ovariectomy and a multipledeficient diet. Am J Pathol 184: 765-777.

43. Weaver CM (2014) Calcium Supplementation: Is Protecting Against Osteoporosis Counter to Protecting against Cardiovascular Disease? Curr Osteoporos Rep.

44. Seale P, Bjork B, Yang W, Kajimura S, Chin S, et al. (2008) PRDM16 controls a brown fat/skeletal muscle switch. Nature 454: 961-967.

45. Gustafson B, Smith U (2010) Activation of canonical wingless-type MMTV integration site family (Wnt) signaling in mature adipocytes increases betacatenin levels and leads to cell dedifferentiation and insulin resistance. J Biol Chem 285: 14031-14041.

46. Gonzalez-Sancho JM, Brennan KR, Castelo-Soccio LA, Brown AM (2004) Wnt proteins induce dishevelled phosphorylation via an LRP5/6- independent mechanism, irrespective of their ability to stabilize beta-catenin. Mol Cell Biol 24: $4757-4768$.

47. Katoh M (2005) WNT/PCP signaling pathway and human cancer (review). Oncol Rep 14: 1583-1588.

48. Katoh M, Katoh M (2007) WNT signaling pathway and stem cell signaling network. Clin Cancer Res 13: 4042-4045.

49. Foo C, Frey S, Yang HH, Zellweger R, Filgueira L (2007) Downregulation of beta-catenin and transdifferentiation of human osteoblasts to adipocytes under estrogen deficiency. Gynecol Endocrinol 23: 535-540. 